\title{
Uplink Narrowband IoT Data Rate Improvement: Dense Modulation Formats or Non-Orthogonal Signal Waveforms?
}

\author{
Tongyang $\mathrm{Xu}$ \\ Department of Electronic and Electrical Engineering \\ University College London \\ Email: tongyang.xu.11@ucl.ac.uk
}

\author{
Izzat Darwazeh \\ Department of Electronic and Electrical Engineering \\ University College London \\ Email: i.darwazeh@ucl.ac.uk
}

\begin{abstract}
Narrowband Internet of Things (NB-IoT) is widely used in low power wide area network (LPWAN) applications due to its long distance coverage and low power consumption. According to the 3GPP NB-IoT standard, the maximum modulation format that NB-IoT can support is QPSK, which limits the data rate sensitive IoT applications. To overcome this limitation either higher order modulation formats such as 8PSK or advanced non-orthogonal signal waveforms can be used. In this work, we propose an enhanced NB-IoT framework, eNB-IoT, which applies a non-orthogonal spectrally efficient frequency division multiplexing (SEFDM) signal waveform beyond the typical orthogonal frequency division multiplexing (OFDM). Since the uplink signal recovery is at base station (BS), in this work we use an efficient and sophisticated minimum Euclidean norm search detector, termed sphere decoding (SD) detector. Simulation results indicate that to achieve the same data rate improvement (i.e. 50\% improvement) and BER performance, eNB-IoT, employing the non-orthogonal signal waveform, requires $3 \mathrm{~dB}$ less transmission power than typical NB-IoT using the dense modulation approach at $\mathrm{BER}=10^{-5}$. The saved power can either extend the battery life of IoT devices or extend the signal transmission distance. This work also proposes an overlapped SD (OSD) detector to simplify the BS signal processing. Its parallel architecture, employing multiple small size SD kernels, can speed up the signal detection. Results show that by using the OSD detector, eNB-IoT still outperforms NB-IoT but with one order of magnitude complexity reduction.
\end{abstract}

Index Terms-Internet of Things, Narrowband, NB-IoT, eNB-IoT, 5G, non-orthogonal, sphere decoding.

\section{INTRODUCTION}

Internet of things (IoT) is changing our environment since more and more devices are connected to provide all kinds of services. In order to have secure and reliable connections for massive IoT devices, narrowband IoT (NB-IoT) [1]-[3], operated in licensed frequency bands, was proposed following long term evolution (LTE) specifications [1]. Thus its deployment is straightforward on existing physical infrastructures.

For long distance IoT communications, signal bandwidth is narrow in order to reduce its noise power within the signal bandwidth. Additional benefits such as enhanced capacity, extended coverage and low power consumption are also introduced due to the narrow bandwidth. NB-IoT occupies one LTE physical resource block (PRB) with 12 sub-carriers and $15 \mathrm{kHz}$ sub-carrier spacing. NB-IoT was initially designed for low data rate IoT applications. However, with the ever-increasing service requirements, data rate increase is becoming critical. The next generation NB-IoT should support various data rate according to different application scenarios. 3GPP release 14 [1] has offered options to include potential modulation and coding scheme (MCS) for the next generation NB-IoT.

Currently, the maximum modulation format supported by NB-IoT is QPSK [1], [4]. One possible solution for its data rate improvement is to employ higher order modulation formats. An alternative approach is to use specially designed signal waveforms. In this work, we focus on a non-orthogonal signal waveform, SEFDM [5], to improve flexibly data rate without upgrading modulation formats. In order to distinguish from the typical NB-IoT, we define a new term, enhanced NB-IoT (eNB-IoT), for our proposed work. Simulation results show that to achieve the same data rate, the proposed eNB-IoT can save $3 \mathrm{~dB}$ signal transmission power than NB-IoT. The saved power budget can either improve battery life of IoT devices or further extend the IoT signal transmission distance.

\section{ENHANCED NB-IOT SignAL WAVEFORM}

The initial idea of the non-orthogonal waveform is to pack sub-carriers closer in frequency, thus signal bandwidth is compressed as shown in Fig. 1, which is "SEFDM Type-I". The benefit of this signal type is to transmit the same amount of data while occupying narrower bandwidth. Thus, noise power is reduced over a narrower signal bandwidth and the number of connected IoT devices can be increased via occupying the saved bandwidth. Furthermore, based on this Type-I signal waveform, a data rate improved "SEFDM TypeII" waveform is proposed as shown in Fig. 1. The key idea of Type-II is to increase data rate per sub-carrier on the Type-I signal. Compared with the OFDM signal 


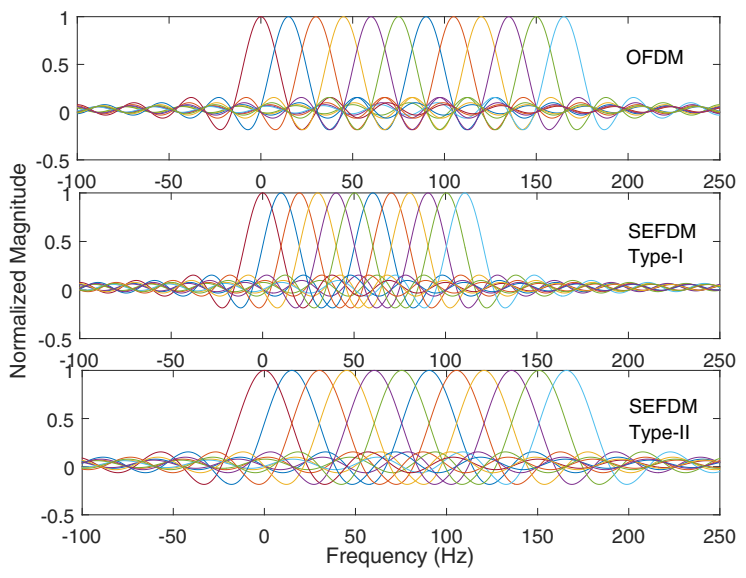

Fig. 1. Sub-carrier allocation schemes for different multicarrier signals. OFDM (12 sub-carriers, data rate is $R_{b}$ ). SEFDM Type-I ( 12 sub-carriers, $\alpha=0.67$, data rate is $R_{b}$ ). SEFDM Type-II (12 sub-carriers, $\alpha=0.67$, data rate is $1.5 R_{b}$ ).

waveform, it is clearly seen that the bandwidth of each sub-carrier of the Type-II signal is wider leading to an improved data rate per sub-carrier and therefore the improved data rate for the entire signal.

The mathematical expression of the $N$ sub-carrier SEFDM signal is defined in matrix form as

$$
X=\mathbf{F} S,
$$

where $X$ is an $N$-dimensional vector of SEFDM time samples, $S$ is an $N$-dimensional vector of transmitted symbols such as QPSK and $\mathbf{F}$ is an $N \times N$ sub-carrier matrix with elements equal to $e^{\frac{j 2 \pi n k \alpha}{N}}$ where $\alpha$ is the bandwidth compression factor.

At the receiver, the signal is contaminated by additive white Gaussian noise (AWGN), denoted as $Z$. Signal demodulation is managed by multiplying with the conjugate sub-carrier matrix $\mathbf{F}^{*}$ with elements equal to $e^{\frac{-j 2 \pi n k \alpha}{N}}$. The demodulation process, effectively a matched filter (MF) detection, is expressed as

$$
R=\mathbf{F}^{*}(X+Z)=\mathbf{F}^{*} \mathbf{F} S+\mathbf{F}^{*} Z=\mathbf{C} S+Z_{\mathbf{F}^{*}},
$$

where $R$ is an $N$-dimensional vector of demodulated symbols, $Z_{\mathbf{F}^{*}}$ is the AWGN correlated with the conjugate sub-carrier matrix and $\mathbf{C}$ is an $N \times N$ correlation matrix equals to $\mathbf{C}=\mathbf{F}^{*} \mathbf{F}$ whose elements are expressed as

$$
\begin{aligned}
c_{m, n} & =\frac{1}{N} \sum_{k=1}^{N} e^{\frac{j 2 \pi m k \alpha}{N}} e^{-\frac{j 2 \pi n k \alpha}{N}} \\
& =\left\{\begin{array}{ll}
1 & , m=n \\
\frac{1-e^{j 2 \pi \alpha(m-n)}}{N\left(1-e^{\frac{j 2 \pi \alpha(m-n)}{N}}\right)} & , m \neq n
\end{array}\right\},
\end{aligned}
$$

where $m, n$ are indices of two arbitrary sub-carriers. The off-diagonal terms $(m \neq n)$ in the correlation matrix $\mathbf{C}$ indicate the inter carrier interference (ICI) caused by non-orthogonal sub-carrier overlapping.

\section{UPLINK Signal DECODING}

The eNB-IoT signal is designed in one PRB and the number of sub-carriers is limited to 12 , which is small enough for robust signal detection algorithms. In addition, the uplink signal decoding is within base stations. Thus, IoT devices would be designed simply to generate signals and all signal detection work will be at the base station. In this case, battery power in each eNB-IoT device would not be consumed too much even applying sophisticated signal detectors.

\section{A. Sphere Decoding}

Due to the loss of orthogonality between sub-carriers, the received signals contain serious ICI leading to increased error rates. Optimal performance can be achieved using an exhaustive search method such as maximum likelihood (ML), which tests all possible combinations of input symbols for every received SEFDM symbols. However, the exponentially increased complexity makes the detection impractical. Therefore, a complexity reducing detection technique termed sphere decoding (SD) [6] was proposed and modified for SEFDM [7]. In contrast, SD algorithm achieves the ML performance by searching for the best solution within a predefined sphere space. Since the defined search space is smaller than the ML search space, SD leads to significantly reduced complexity. The search for the best estimate $S_{S D}$ in SEFDM is defined as

$$
S_{S D}=\arg \min _{S \in O^{N}}\|R-\mathbf{C} S\|^{2} \leq g,
$$

where $\|$.$\| denotes Euclidean norm, O$ is constellation cardinality and $O^{N}$ is the set of all possible symbols combinations. The optimum solution is the one that has the smallest Euclidean norm within the predefined initial radius $g$, which equals the distance between the demodulated symbols $R$ and the initial constrained estimate $S_{Z F}$ where $S_{Z F}$ is the zero forcing estimate which can be obtained using the rounding function $\lfloor .7$ as $S_{Z F}=\left\lfloor\mathbf{C}^{-1} R\right\rceil$. The initial radius is expressed as

$$
g=\left\|R-\mathbf{C} S_{Z F}\right\|^{2} .
$$

By substituting $P=\mathbf{C}^{-1} R$ and using Cholesky decomposition $\operatorname{chol}\left\{\mathbf{C}^{*} \mathbf{C}\right\}=\mathbf{L}^{*} \mathbf{L}$, (4) and (5) can be transformed to (6) and (7), respectively.

$$
\begin{gathered}
S_{S D}=\arg \min _{S \in O^{N}}\|\mathbf{L}(P-S)\|^{2} \leq g . \\
g=\left\|\mathbf{L}\left(P-S_{Z F}\right)\right\|^{2} .
\end{gathered}
$$

In order to investigate each square term in (6), the inequality expression for the $N^{t h}$ term is represented as

$$
l_{N, N}^{2}\left(P_{N}-S_{N}\right)^{2} \leq g_{N}=g .
$$


Therefore, the search interval for the $N^{t h}$ symbol is expressed in (9), (10), (11)

$$
\begin{gathered}
L B \leq S_{N} \leq U B, \\
L B=\left\lceil-\frac{\sqrt{g_{N}}}{l_{N, N}}+P_{N}\right\rceil, \\
U B=\left\lfloor\frac{\sqrt{g_{N}}}{l_{N, N}}+P_{N}\right\rfloor,
\end{gathered}
$$

where the $\lceil\cdot\rceil$ and $\lfloor\cdot\rfloor$ denote rounding operation to the nearest larger and smaller integer, respectively. Assume there is a solution $S_{N}$ in this interval, the radius is updated as

$$
g_{N-1}=g_{N}-l_{N, N}^{2}\left(P_{N}-S_{N}\right)^{2} .
$$

The algorithm employes the similar enumeration scheme in (9) to find possible solutions in the $(N-1)^{t h}$ dimension. The same process is iterated until the last dimension. When SD reaches the last dimension $i=1$, the solution is defined as a $\mathrm{N}$-dimensional vector that meets the initial radius constraint.

\section{B. Overlapped Sphere Decoding}

It is apparent from (4) that the computational complexity of the SD detection depends on the size of the correlation matrix $\mathbf{C}$, which is associated with the number of sub-carriers. In order to reduce the complexity, we appropriately adapt the flexible matrix transformation method, originally proposed in [8] for equalization of time-variant channel in OFDM, to decompose the entire matrix $\mathbf{C}$ into multiple sub-matrices and operate SD detection for each.

By studying the matrix $\mathbf{C}$ in (3), it is noted that $\mathbf{C}$ is a sparse matrix where most of the energy is concentrated in the diagonal elements and their closest neighbouring non-diagonal elements. Since $\mathbf{C}$ is a sparse matrix, thus it is not efficient to perform signal detection based on the entire $\mathbf{C}$.

$$
\mathbf{C}=\left[\begin{array}{cccc}
c_{1,1} & c_{1,2} & \cdots & c_{1, N} \\
c_{2,1} & c_{2,2} & \ddots & \vdots \\
\vdots & \ddots & \ddots & c_{N-1, N} \\
c_{N, 1} & \cdots & c_{N, N-1} & c_{N, N}
\end{array}\right] .
$$

An efficient way is to transform the $N \times N$ matrix $\mathbf{C}$ into a new band matrix $\mathbb{C}$ by deriving from multiple and overlapped block diagonal matrices $\mathbf{G}_{\mathbf{n}}$ (termed submatrices) each of size $q \times q$. Hence, the matrix $\mathbf{C}$ is converted to a new format as shown in (14).

$$
\mathbb{C}=\left[\begin{array}{cccc}
\mathbf{G}_{1} & 0 & \cdots & 0 \\
0 & \mathbf{G}_{2} & \cdots & 0 \\
\vdots & \vdots & \ddots & \vdots \\
0 & 0 & \cdots & \mathbf{G}_{\mathbf{N}-\mathbf{q}+\mathbf{1}}
\end{array}\right]
$$

where the sub-matrix $\mathbf{G}_{\mathbf{n}}$ is expressed as

$$
\mathbf{G}_{\mathbf{n}}=\left[\begin{array}{ccc}
c_{n, n} & \cdots & c_{n, n+q-1} \\
c_{n+1, n} & \ddots & \vdots \\
\vdots & \ddots & c_{n+q-2, n+q-1} \\
c_{n+q-1, n} & \cdots & c_{n+q-1, n+q-1}
\end{array}\right] .
$$

Therefore, the size of the matrix $\mathbb{C}$ in (14) is $\mathcal{N} \times \mathcal{N}$ where $\mathcal{N}=(N-q+1) \times q$. In order to realize signal detection, the demodulated symbol vector $R$ is converted to a new format as

$$
R \Rightarrow \mathcal{R}=\left[B_{1}, B_{2}, \ldots, B_{N-q+1}\right],
$$

where $B_{n}$ is a $q$-dimensional vector includes elements

$$
B_{n}=[R(n), R(n+1), \ldots, R(n+q-1)] .
$$

Therefore, $\mathcal{R}$ is a $\mathcal{N}$-dimensional vector. The typical signal equalization to $\mathcal{R}$ is operated as

$$
\mathcal{R}_{e q}=\mathbb{C}^{-1} \mathcal{R}
$$

where

$$
\mathbb{C}^{-1}=\left[\begin{array}{cccc}
\mathbf{G}_{\mathbf{1}}^{-1} & 0 & \cdots & 0 \\
0 & \mathbf{G}_{\mathbf{2}}^{-1} & \cdots & 0 \\
\vdots & \vdots & \ddots & \vdots \\
0 & 0 & \cdots & \mathbf{G}_{\mathbf{N}-\mathbf{q}+\mathbf{1}}^{-\mathbf{1}}
\end{array}\right] .
$$

It is clearly seen that due to the diagonal characteristic of the matrix $\mathbb{C}$, its matrix inverse can be obtained from the inverse of each sub-matrix, indicating reduced computational complexity. Therefore, (18) can be further simplified to

$$
\mathcal{R}_{n}=\mathbf{G}_{\mathbf{n}}^{-1} B_{n},
$$

where $n \in[1, \ldots, N-q+1]$. It is evident that the size of the multiplication is reduced indicating reduced computational complexity. We assume $q$ is an odd number in this work. For each multiplication in (20) with $n \in[2, \ldots, N-q]$, the middle element (i.e. $(q+1) / 2)$ is selected as one estimated symbol. For the first submatrix multiplication (i.e. $n=1$ ) and the last one (i.e. $n=N-q+1)$, the first $(q+1) / 2$ and the last $(q+1) / 2$ elements are selected, respectively. Therefore, the estimated symbol vector $R_{e q}$ of size $N$ is expressed as

$$
\begin{aligned}
R_{e q}= & {\left[\mathcal{R}_{1}(1), \ldots, \mathcal{R}_{1}((q+1) / 2), \mathcal{R}_{2}((q+1) / 2), \ldots,\right.} \\
& \mathcal{R}_{N-q}((q+1) / 2), \mathcal{R}_{N-q+1}((q+1) / 2), \ldots, \\
& \left.\mathcal{R}_{N-q+1}(q)\right]
\end{aligned}
$$

It indicates that the $S_{Z F}$ calculation in (5) and $P$ calculation in (6) can be greatly simplified using the principle derived from (20). In addition, the computation complexity of (4) can be reduced as well to a system size constrained by the sub-matrix $\mathbf{G}_{\mathbf{n}}$. 


\section{Performance Comparisons}

The typical NB-IoT and our proposed eNB-IoT signal waveforms are compared in terms of spectral efficiency (SE) and data rate improvement in Table I. For the typical NB-IoT, the maximum supported modulation format by 3GPP is QPSK. In order to show the data improvement by using higher order modulation format, 8PSK is evaluated for NB-IoT. For our proposed eNBIoT signals, only QPSK is used. In general, a higher spectral efficiency value, a higher data rate can be achieved. The single SD detector in section III-A is noted as "SD Type-I" and the overlapped SD (OSD) detector in section III-B is noted as "SD Type-II".

Table I: Spectral efficiency and corresponding data rate

\begin{tabular}{lll}
\hline Parameters & SE $(\mathrm{bit} / \mathrm{s} / \mathrm{Hz})$ & Data rate \\
\hline \hline NB-IoT & $2(\mathrm{QPSK})$ & $R_{b}$ \\
\hline eNB-IoT & $2.5(\mathrm{QPSK})$ & $1.25 R_{b}(+25 \%)$ \\
\hline NB-IoT & $3(8 \mathrm{PSK})$ & $1.5 R_{b}(+50 \%)$ \\
\hline eNB-IoT & 3 (QPSK) & $1.5 R_{b}(+50 \%)$ \\
\hline
\end{tabular}

First, the SD Type-I detectors for spectral efficiency values equal to $2.5 \mathrm{bit} / \mathrm{s} / \mathrm{Hz}$ and $3 \mathrm{bit} / \mathrm{s} / \mathrm{Hz}$ are evaluated in AWGN channel in Fig. 2(a) and Fig. 2(b), respectively. Both figures show that due to the ICI in eNB-IoT (SEFDM) signals, typical MF detectors can not effectively recover signals. In Fig. 2(a), by using SD Type-I detector, the eNB-IoT signal with $2.5 \mathrm{bit} / \mathrm{s} / \mathrm{Hz}$ spectral efficiency can achieve the identical performance as the typical NB-IoT signal with $2 \mathrm{bit} / \mathrm{s} / \mathrm{Hz}$ spectral efficiency. It indicates that $25 \%$ data rate is improved with no performance loss. In Fig. 2(b), considering 3 $\mathrm{bit} / \mathrm{s} / \mathrm{Hz}$ spectral efficiency, the eNB-IoT signal with QPSK modulation outperforms the typical NB-IoT with $8 \mathrm{PSK}$ by $3 \mathrm{~dB}$ at $\mathrm{BER}=10^{-5}$. It indicates that in order to achieve higher data rates, the non-orthogonal signal waveform is more efficient than the higher order modulation scheme. ${ }^{1}$

The effect of $q \times q$ sub-matrix is illustrated in Fig. 3 . In Fig. 3(a), using the $3 \times 3$ sub-matrix, the BER performance of eNB-IoT is far away from the NB-IoT result. Increasing the sub-matrix size to $9 \times 9$, the BER curve approaches the NB-IoT one with narrow performance gap. By increasing the sub-matrix size to $11 \times 11$, the performance is similar to the NB-IoT. It is concluded that optimal performance can be achieved with reduced complexity by using the SD Type-II detector. The same results are observed in Fig. 3(b).

\section{Detector Complexity Analysis}

In order to simplify the computation, in this section, we use the number of visited nodes within OSD to

\footnotetext{
${ }^{1}$ It should be noted that since the typical NB-IoT employs orthogonal signals and no ICI exists, the use of SD or MF detectors on QPSK/8PSK modulated signals offers no performance advantage.
}
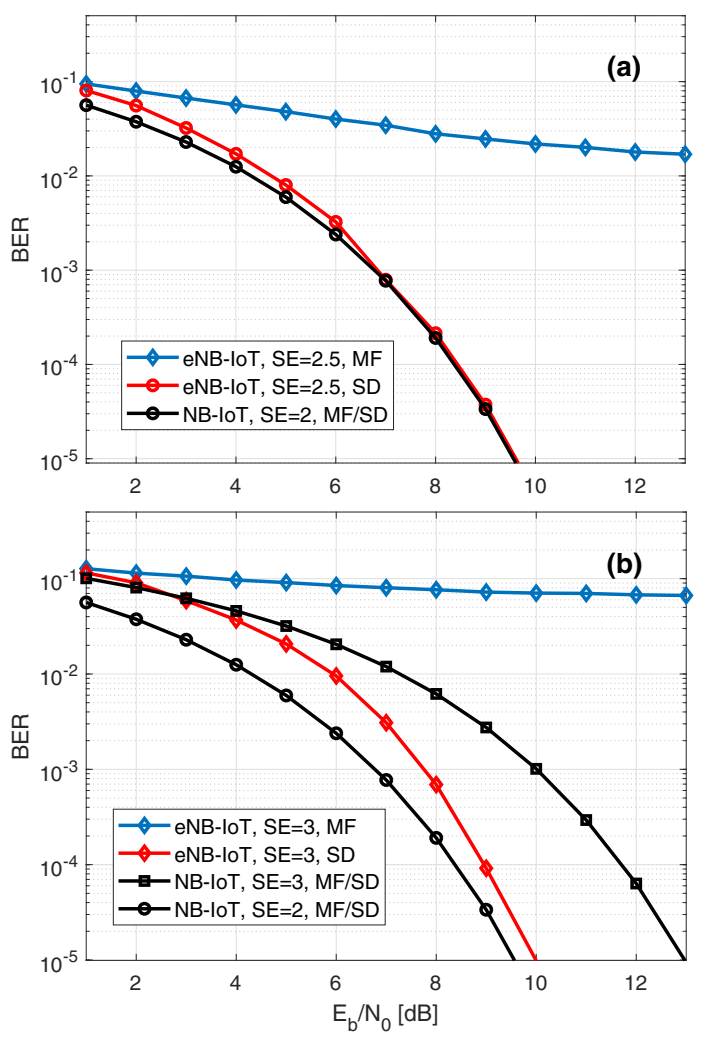

Fig. 2. Performance of SD Type-I detector for QPSK modulated eNB-IoT signals (a) $\alpha=0.8$; (b) $\alpha=0.67$, and QPSK, 8PSK modulated NB-IoT signals.

estimate the complexity. In addition, due to the random complexity of sphere decoding, all the nodes are assumed to be visited in the calculation. Therefore, the number of visited nodes at each layer is defined as $O^{v}$, where $v$ indicates the searching layer index with the range $[1,2, \ldots q]$. Depending on the size (i.e. $q$ ) and the number (i.e. $N_{b}$ ) of OSD sub-matrices, the total number of visited nodes are defined as

$$
N_{v}=N_{b} \times \frac{O\left(1-O^{q}\right)}{1-O} .
$$

The total visited nodes and BER performance are shown together in Fig. 4. It is clearly seen that with the increase of each OSD sub-matrix size $q$, the visited nodes are increased significantly. BER values at $E_{b} / N_{o}=10 \mathrm{~dB}$ for both NB-IoT and eNB-IoT are included for comparisons. Since there is no need to use sphere decoding for NB-IoT, therefore, its performance is fixed at all sub-matrix sizes. However, for eNBIoT, a bigger sub-matrix size leads to improved performance (i.e. smaller BER). An important discovery is that when $q=9$ is defined, eNB-IoT starts to achieve better performance than the typical NB-IoT. This discovery shows a trade-off between BER performance and design complexity. The eNB-IoT signals with both SD Type- 

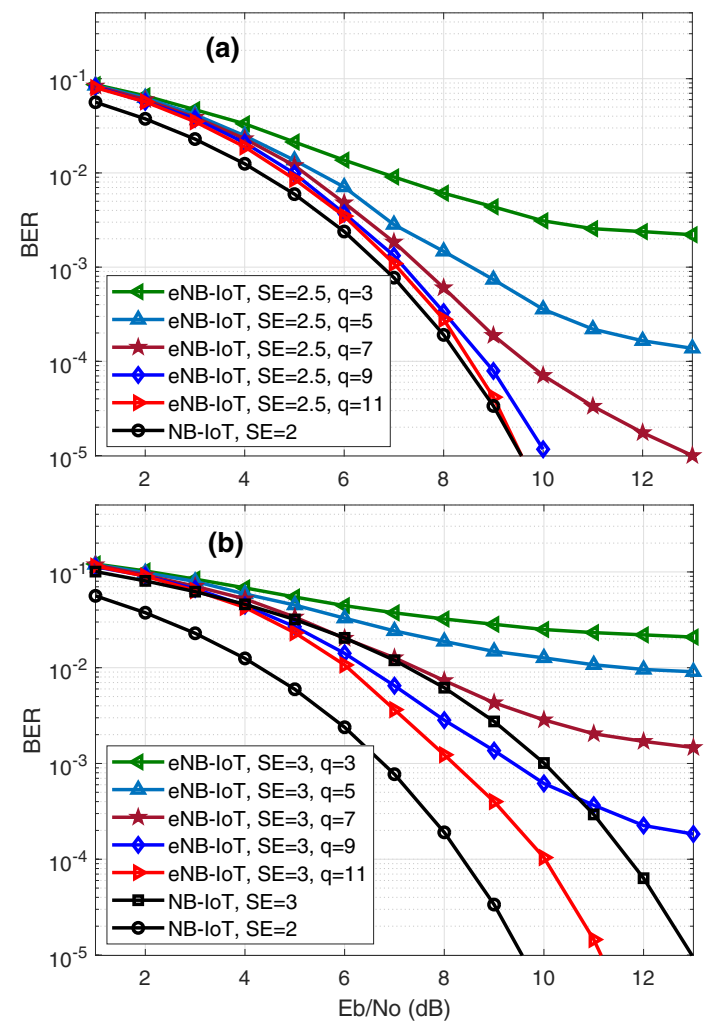

Fig. 3. Performance of SD Type-II detector with different submatrix sizes.

I $(q=12)$ and SD Type-II $(q=9)$ perform better than the NB-IoT, but the SD Type-II $(q=9)$ is one order of magnitude complexity lower than the SD Type-I $(q=12)$. In practice, since base station will perform the signal detection, thus, the power consumption of each IoT device doesn't change too much.

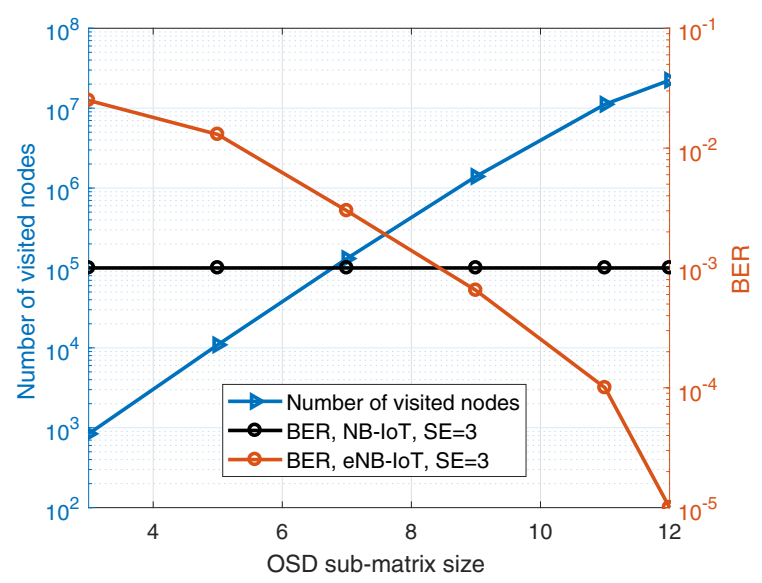

Fig. 4. The number of visited nodes and BER $(\mathrm{Eb} / \mathrm{No}=10 \mathrm{~dB})$ performance versus OSD sub-matrix size.

\section{CONCLusions}

This work aims to design an efficient solution to speed up the uplink signal transmission for next generation NB-IoT. The maximum supported modulation format defined by 3GPP is QPSK. One straightforward solution for data improvement is to use higher order modulation formats such as 8PSK. This work proposes an alternative solution; to use an advanced signal waveform to achieve the same target. We considered a bandwidth compressed waveform termed SEFDM, which saves bandwidth by packing sub-carriers closer. Based on this idea, an SEFDM Type-II signal was designed by improving data rate on each sub-carrier. Thus, the total data rate is improved without occupying extra bandwidth. Since signal processing pressure of uplink signals is at the base station, thus the sophisticated minimum Euclidean norm search SD detector is applied to guarantee the error rate performance. Results show that without modulation format upgrade, the proposed signal waveform can achieve $25 \%$ data rate improvement with no performance loss. Furthermore, with $50 \%$ data rate improvement, the proposed QPSK modulated eNBIoT signal outperforms the equivalent 8PSK NB-IoT signals by $3 \mathrm{~dB}$ at $\mathrm{BER}=10^{-5}$. It proves that the advanced signal waveform performs more efficiently than dense modulation formats in data rate improvement. In addition, a parallel overlapped sphere decoding (OSD) detector, which partitions the signal detection work into multiple small blocks, is proposed and tested to simplify the typical single SD detector. Results show that the performance gain still exists with complexity reduced by one order of magnitude.

\section{REFERENCES}

[1] 3GPP TS 36.213 v.14.2.0, "LTE; evolved universal terrestrial radio access (E-UTRA); physical layer procedures," Rel. 14, Apr. 2017.

[2] J. Xu, J. Yao, L. Wang, Z. Ming, K. Wu, and L. Chen, "Narrowband Internet of Things: Evolutions, technologies and open issues," IEEE Internet of Things Journal, vol. PP, no. 99, pp. 1-1, 2017.

[3] T. Xu and I. Darwazeh, "Non-orthogonal narrowband Internet of Things: A design for saving bandwidth and doubling the number of connected devices," IEEE Internet of Things Journal, vol. 5, no. 3, pp. 2120-2129, June 2018.

[4] M. Chen, Y. Miao, Y. Hao, and K. Hwang, "Narrow band Internet of Things," IEEE Access, vol. 5, pp. 20 557-20 577, 2017.

[5] T. Xu and I. Darwazeh, "Transmission experiment of bandwidth compressed carrier aggregation in a realistic fading channel," IEEE Transactions on Vehicular Technology, vol. 66, no. 5, pp. 40874097, May 2017.

[6] B. Hassibi and H. Vikalo, "On the sphere-decoding algorithm I. expected complexity," IEEE Transactions on Signal Processing, vol. 53, no. 8, pp. 2806-2818, Aug 2005.

[7] I. Kanaras, A. Chorti, M. Rodrigues, and I. Darwazeh, "A fast constrained sphere decoder for ill conditioned communication systems," IEEE Communications Letters, vol. 14, no. 11, pp. 9991001, November 2010.

[8] W. G. Jeon, K. H. Chang, and Y. S. Cho, "An equalization technique for orthogonal frequency-division multiplexing systems in time-variant multipath channels," IEEE Transactions on Communications, vol. 47, no. 1, pp. 27-32, Jan 1999. 